Electronic Supporting Information (J. Chem. Eng. Data)

\title{
Density Measurements under Pressure for the Binary System 1-Propanol + Toluene
}

\section{Claus K. Zéberg-Mikkelsen*" and Simon I. Andersen}

Center for Phase Equilibria and Separation Processes (IVCSEP), Department of Chemical Engineering, Technical University of Denmark, Building 229, 2800 Lyngby, Denmark

* Corresponding author. Phone: (-45) 452528 00; Fax: (-45) 8822 58; E-mail: czm@kt.dtu.dk

Table 2. Estimated isothermal compressibility $\kappa_{T}$ for 1-propanol (1) + toluene (2) mixtures versus temperature $T$, pressure $P$, and mole fraction $x_{1}$.

\begin{tabular}{|c|c|c|c|c|c|c|c|}
\hline \multirow[b]{2}{*}{$T / \mathrm{K}$} & \multirow[b]{2}{*}{$P / \mathrm{MPa}$} & $x_{1}=0$ & $x_{1}=0.200$ & $x_{1}=0.400$ & $x_{1}=0.600$ & $x_{1}=0.800$ & $x_{1}=1$ \\
\hline & & \multicolumn{6}{|c|}{$10^{4} \cdot \kappa_{T} / \mathrm{MPa}^{-1}$} \\
\hline 303.15 & 0.1 & 9.44 & 9.69 & 9.83 & 9.94 & 10.18 & 10.33 \\
\hline 303.15 & 5 & 9.00 & 9.23 & 9.35 & 9.44 & 9.63 & 9.81 \\
\hline 303.15 & 10 & 8.60 & 8.79 & 8.90 & 8.99 & 9.13 & 9.33 \\
\hline 303.15 & 15 & 8.23 & 8.40 & 8.50 & 8.57 & 8.69 & 8.90 \\
\hline 303.15 & 20 & 7.90 & 8.05 & 8.13 & 8.20 & 8.29 & 8.50 \\
\hline 303.15 & 25 & 7.59 & 7.72 & 7.80 & 7.86 & 7.92 & 8.15 \\
\hline 303.15 & 30 & 7.31 & 7.42 & 7.49 & 7.55 & 7.59 & 7.82 \\
\hline 313.15 & 0.1 & 10.00 & 10.32 & 10.35 & 10.43 & 10.73 & 10.86 \\
\hline 313.15 & 5 & 9.54 & 9.81 & 9.86 & 9.95 & 10.17 & 10.31 \\
\hline 313.15 & 10 & 9.12 & 9.35 & 9.41 & 9.49 & 9.65 & 9.80 \\
\hline 313.15 & 15 & 8.73 & 8.93 & 9.01 & 9.08 & 9.18 & 9.35 \\
\hline 313.15 & 20 & 8.38 & 8.55 & 8.63 & 8.71 & 8.76 & 8.94 \\
\hline 313.15 & 25 & 8.06 & 8.20 & 8.29 & 8.37 & 8.38 & 8.56 \\
\hline 313.15 & 30 & 7.76 & 7.88 & 7.98 & 8.05 & 8.03 & 8.21 \\
\hline 323.15 & 0.1 & 10.72 & 11.12 & 11.33 & 11.32 & 11.59 & 11.67 \\
\hline 323.15 & 5 & 10.21 & 10.54 & 10.72 & 10.75 & 10.95 & 11.04 \\
\hline 323.15 & 10 & 9.75 & 10.02 & 10.16 & 10.23 & 10.36 & 10.48 \\
\hline 323.15 & 15 & 9.32 & 9.54 & 9.67 & 9.77 & 9.84 & 9.97 \\
\hline 323.15 & 20 & 8.94 & 9.11 & 9.22 & 9.34 & 9.37 & 9.51 \\
\hline 323.15 & 25 & 8.58 & 8.72 & 8.81 & 8.96 & 8.94 & 9.09 \\
\hline 323.15 & 30 & 8.26 & 8.37 & 8.44 & 8.60 & 8.56 & 8.71 \\
\hline 333.15 & 0.1 & 11.59 & 11.92 & 12.17 & 12.22 & 12.38 & 12.52 \\
\hline 333.15 & 5 & 10.98 & 11.27 & 11.50 & 11.56 & 11.68 & 11.80 \\
\hline 333.15 & 10 & 10.43 & 10.69 & 10.89 & 10.96 & 11.05 & 11.15 \\
\hline 333.15 & 15 & 9.93 & 10.17 & 10.34 & 10.42 & 10.48 & 10.57 \\
\hline 333.15 & 20 & 9.47 & 9.69 & 9.85 & 9.93 & 9.97 & 10.05 \\
\hline 333.15 & 25 & 9.06 & 9.27 & 9.41 & 9.49 & 9.51 & 9.58 \\
\hline 333.15 & 30 & 8.69 & 8.87 & 9.01 & 9.09 & 9.10 & 9.16 \\
\hline
\end{tabular}


Table 3. Estimated isobaric thermal expansivity $\alpha_{P}$ for 1-propanol (1) + toluene (2) mixtures versus temperature $T$, pressure $P$, and mole fraction $x_{1}$.

\begin{tabular}{|c|c|c|c|c|c|c|c|}
\hline \multirow[b]{2}{*}{$T / \mathrm{K}$} & \multirow[b]{2}{*}{$P / \mathrm{MPa}$} & $x_{1}=0$ & $x_{1}=0.200$ & $x_{1}=0.400$ & $x_{1}=0.600$ & $x_{1}=0.800$ & $x_{1}=1$ \\
\hline & & \multicolumn{6}{|c|}{$10^{4} \cdot \alpha_{P} / \mathrm{K}^{-1}$} \\
\hline 303.15 & 0.1 & 10.99 & 11.32 & 11.45 & 11.32 & 11.02 & 10.48 \\
\hline 303.15 & 5 & 10.70 & 11.02 & 11.11 & 10.99 & 10.70 & 10.18 \\
\hline 303.15 & 10 & 10.35 & 10.66 & 10.75 & 10.64 & 10.37 & 9.85 \\
\hline 303.15 & 15 & 10.05 & 10.35 & 10.44 & 10.32 & 10.05 & 9.55 \\
\hline 303.15 & 20 & 9.79 & 10.08 & 10.15 & 10.03 & 9.76 & 9.29 \\
\hline 303.15 & 25 & 9.55 & 9.83 & 9.88 & 9.75 & 9.50 & 9.04 \\
\hline 303.15 & 30 & 9.32 & 9.59 & 9.63 & 9.51 & 9.26 & 8.83 \\
\hline 313.15 & 0.1 & 11.11 & 11.45 & 11.58 & 11.44 & 11.14 & 10.59 \\
\hline 313.15 & 5 & 10.82 & 11.14 & 11.24 & 11.11 & 10.82 & 10.28 \\
\hline 313.15 & 10 & 10.46 & 10.78 & 10.87 & 10.75 & 10.47 & 9.95 \\
\hline 313.15 & 15 & 10.16 & 10.46 & 10.55 & 10.43 & 10.15 & 9.64 \\
\hline 313.15 & 20 & 9.89 & 10.18 & 10.25 & 10.13 & 9.85 & 9.37 \\
\hline 313.15 & 25 & 9.64 & 9.93 & 9.98 & 9.85 & 9.59 & 9.12 \\
\hline 313.15 & 30 & 9.41 & 9.68 & 9.72 & 9.60 & 9.35 & 8.90 \\
\hline 323.15 & 0.1 & 11.23 & 11.58 & 11.72 & 11.58 & 11.26 & 10.70 \\
\hline 323.15 & 5 & 10.94 & 11.27 & 11.36 & 11.24 & 10.93 & 10.39 \\
\hline 323.15 & 10 & 10.57 & 10.89 & 10.99 & 10.87 & 10.58 & 10.05 \\
\hline 323.15 & 15 & 10.26 & 10.57 & 10.66 & 10.54 & 10.25 & 9.73 \\
\hline 323.15 & 20 & 9.99 & 10.29 & 10.36 & 10.23 & 9.95 & 9.46 \\
\hline 323.15 & 25 & 9.74 & 10.03 & 10.08 & 9.95 & 9.68 & 9.21 \\
\hline 323.15 & 30 & 9.50 & 9.78 & 9.82 & 9.69 & 9.44 & 8.98 \\
\hline 333.15 & 0.1 & 11.36 & 11.72 & 11.86 & 11.71 & 11.39 & 10.82 \\
\hline 333.15 & 5 & 11.06 & 11.40 & 11.50 & 11.37 & 11.06 & 10.50 \\
\hline 333.15 & 10 & 10.68 & 11.01 & 11.11 & 10.99 & 10.70 & 10.15 \\
\hline 333.15 & 15 & 10.37 & 10.68 & 10.78 & 10.65 & 10.36 & 9.83 \\
\hline 333.15 & 20 & 10.09 & 10.40 & 10.47 & 10.34 & 10.05 & 9.55 \\
\hline 333.15 & 25 & 9.83 & 10.13 & 10.18 & 10.05 & 9.78 & 9.30 \\
\hline 333.15 & 30 & 9.59 & 9.87 & 9.92 & 9.79 & 9.53 & 9.07 \\
\hline
\end{tabular}


Table 4. Estimated excess molar volume $v^{E}$ for 1-propanol (1) + toluene (2) mixtures versus temperature $T$, pressure $P$, and mole fraction $x_{1}$.

\begin{tabular}{|c|c|c|c|c|c|c|c|}
\hline \multirow[b]{2}{*}{$T / \mathrm{K}$} & \multirow[b]{2}{*}{$P / \mathrm{MPa}$} & $x_{1}=0$ & $x_{1}=0.200$ & $X_{1}=0.400$ & $x_{1}=0.600$ & $x_{1}=0.800$ & $x_{1}=1$ \\
\hline & & \multicolumn{6}{|c|}{$v^{E} / \mathrm{cm}^{3} \cdot \mathrm{mol}^{-1}$} \\
\hline 303.15 & 0.1 & 0 & 0.0801 & 0.0496 & -0.0264 & -0.0606 & 0 \\
\hline 303.15 & 5 & 0 & 0.0728 & 0.0482 & -0.0251 & -0.0611 & 0 \\
\hline 303.15 & 10 & 0 & 0.0683 & 0.0425 & -0.0260 & -0.0627 & 0 \\
\hline 303.15 & 15 & 0 & 0.0652 & 0.0376 & -0.0293 & -0.0618 & 0 \\
\hline 303.15 & 20 & 0 & 0.0612 & 0.0360 & -0.0277 & -0.0582 & 0 \\
\hline 303.15 & 25 & 0 & 0.0582 & 0.0320 & -0.0284 & -0.0567 & 0 \\
\hline 303.15 & 30 & 0 & 0.0557 & 0.0327 & -0.0258 & -0.0538 & $\begin{array}{l}0 \\
0\end{array}$ \\
\hline 313.15 & 0.1 & 0 & 0.1147 & 0.0954 & 0.0127 & -0.0346 & 0 \\
\hline 313.15 & 5 & 0 & 0.1030 & 0.0921 & 0.0104 & -0.0366 & 0 \\
\hline 313.15 & 10 & 0 & 0.0975 & 0.0875 & 0.0135 & -0.0384 & 0 \\
\hline 313.15 & 15 & 0 & 0.0901 & 0.0827 & 0.0099 & -0.0396 & 0 \\
\hline 313.15 & 20 & 0 & 0.0868 & 0.0788 & 0.0077 & -0.0374 & 0 \\
\hline 313.15 & 25 & 0 & 0.0800 & 0.0756 & 0.0063 & -0.0370 & 0 \\
\hline 313.15 & 30 & 0 & 0.0778 & 0.0760 & 0.0049 & -0.0340 & $\begin{array}{l}0 \\
0\end{array}$ \\
\hline 323.15 & 0.1 & 0 & 0.1610 & 0.1659 & 0.0757 & 0.0065 & 0 \\
\hline 323.15 & 5 & 0 & 0.1433 & 0.1489 & 0.0710 & 0.0003 & 0 \\
\hline 323.15 & 10 & 0 & 0.1350 & 0.1400 & 0.0654 & -0.0022 & 0 \\
\hline 323.15 & 15 & 0 & 0.1294 & 0.1305 & 0.0594 & -0.0059 & 0 \\
\hline 323.15 & 20 & 0 & 0.1255 & 0.1267 & 0.0547 & -0.0071 & 0 \\
\hline 323.15 & 25 & 0 & 0.1220 & 0.1235 & 0.0492 & -0.0074 & 0 \\
\hline 323.15 & 30 & $\begin{array}{l}0 \\
0\end{array}$ & 0.1166 & 0.1188 & 0.0440 & -0.0048 & $\begin{array}{l}0 \\
0\end{array}$ \\
\hline 333.15 & 0.1 & 0 & 0.2157 & 0.2404 & 0.1373 & 0.0412 & 0 \\
\hline 333.15 & 5 & 0 & 0.2028 & 0.2248 & 0.1281 & 0.0358 & 0 \\
\hline 333.15 & 10 & 0 & 0.1960 & 0.2140 & 0.1239 & 0.0329 & 0 \\
\hline 333.15 & 15 & 0 & 0.1868 & 0.2037 & 0.1153 & 0.0296 & 0 \\
\hline 333.15 & 20 & 0 & 0.1783 & 0.1920 & 0.1064 & 0.0253 & 0 \\
\hline 333.15 & 25 & 0 & 0.1719 & 0.1785 & 0.0979 & 0.0228 & 0 \\
\hline 333.15 & 30 & 0 & 0.1649 & 0.1725 & 0.0956 & 0.0208 & 0 \\
\hline
\end{tabular}

